Article

\title{
Evolution of Access Network Sharing and its Role in 5G Networks
}

\author{
Nima Afraz, Frank Slyne, Harleen Gill and Marco Ruffini \\ CONNECT centre, The University of Dublin, Trinity College, Ireland \\ * Correspondence: nafraz@tcd.ie, marco.ruffini@tcd.ie
}

\begin{abstract}
This paper details the evolution of access network sharing models from legacy DSL to the most recent fibre-based technology and the main challenges faced from a technical and business perspectives. We first give an overview of existing access sharing models, that span physical local loop unbundling and virtual unbundled local access. We then describe different types of optical access technologies and highlight how they support network sharing. Next, we examine how the concept of SDN and network virtualization has been pivotal in enabling the idea of "true multi-tenancy", through the use of programmability, flexible architecture and resource isolation. We give examples of recent developments of cloud central office and OLT virtualization. Finally, we provide an insight into the role that novel business models, such as blockchain and smart contract technology, could play in 5G networks. We discuss how these might evolve, to provide flexibility and dynamic operations that are needed in the data and control planes.
\end{abstract}

Keywords: access networks; network sharing; 5G networks; multi tenancy; optical access; sharing economics

\section{Introduction}

By its nature, a telecommunications network is a shared resources that interconnects multiple nodes. Network sharing is part of a fundamental principle of statistical multiplexing of link capacity. Regardless of whether the nodes are setting up connections that reserve capacity in the Plain Old Telephone Service (POTS), or sending packets in a connection-less packet switched network, the overall link capacity is only a fraction of the total interconnection capacity required if all nodes attempted communicating at once. Network sharing also applies to the progressive aggregation of link capacity where the ratio of multiplexing increases in moving from the access towards the core. From the mid-90s', the concept of sharing was extended to also cover the multi-tenant use of the network, where third party network operators compete with the incumbent national operator, so that the same common infrastructure is shared across multiple competing entities. The degree to which infrastructure is shared is limited, on the one hand by physical and logical boundaries that separate resources, and on the other hand by economic complexities such as settlements, agreements and regulations that complicate the sharing process.

Recently, technology evolutions based on Software Defined Network (SDN) and Network Function Virtualization (NFV) have enabled network multi-tenancy that increases the flexibility and level of network control automation and management processes, in ways that were not possible before. These characteristics arise because of virtualization, which enables different entities to get access to a subset of the network resources, while giving the illusion of fully owning that part of the infrastructure. This separates the operations of one tenant fully from other tenants that share the same physical infrastructure. 
$5 \mathrm{G}$ networks are designed to provide higher capacity and to improve performance metrics such as latency, packet loss and availability. The corresponding increase in infrastructure cost requires the network to be shared efficiently across many services and tenant operators. Densification of access points and the virtualisation of the access network has thus become a fundamental principle in the design of $5 \mathrm{G}$ networks. In addition, the growth in infrastructure investment for the $5 \mathrm{G}$ networks is challenging the conventional standalone network ownership model. Operators [1] can save between 20 and 55\% in CapEx by sharing their assets, depending upon how much infrastructure is shared. The 5G Infrastructure Public-Private Partnership (5G PPP) [2] believes that new resource sharing business models are the key enablers for the success of $5 \mathrm{G}$.

This paper is organized as follows. In section 2, we provide a brief history of the development of sharing models for access networks, from early telephone to current fiber-based broadband networks. In section 3, we focus on the challenges for sharing optical access networks, in particular discussing Passive Optical Networks (PONs), showing the importance of network virtualization. Section 4 introduces and elaborates on the applications of SDN and virtualization technologies to realize multi-service, multi-tenant access networks. In section 5 , we study the emerging network ownership models to support network sharing for $5 \mathrm{G}$ and the potential challenges in their design. Finally, we conclude the paper in section 6 .

\section{Access Sharing Models}

Sharing of access networks can be achieved through a number of different architectures and models. Typically there is a trade-off between the ability of Other Licensed Operators (OLOs) to control the level of service offered to their users and the complexity and amount of infrastructure that each OLO needs to deploy. For example, physical layer unbundling provides OLOs with the highest level of control, as they can tap directly into the end-user's physical transmission link, that is, in the case of copper-based Digital Subscriber Line (DSL) access. However, it requires that OLOs to have their own physical infrastructure deployed in (or near) each local Central Office (CO) where they intend to serve customers.

On the other hand, higher layer unbundling, like bitstream access, discussed in more details later, allows OLOs to collect their customer traffic at a small number of points of presence (POPs), thus reducing the amount of equipment deployed in the network. However, these models provide OLOs with very limited control over the type of services they can deliver to the users, as typically they can only offer a small number of broadband packages to their customers. The next sections delve into the details of the most popular access network sharing paradigms.

\subsection{Local Loop Unbundling (LLU)}

Local Loop Unbundling (LLU) [3] was one of the first techniques for enabling competition in the fixed access networks, where the incumbent leases to registered competitors the copper line (called the local loop) connecting the end-user to the local CO . There are three types of LLU, namely: line sharing, full unbundling, and legacy bitstream access.

\subsubsection{Line Sharing}

In line sharing, shown in Fig. 1a, the incumbent operator provides some services to the subscriber while maintaining control over the copper pair. New entrants (i.e., OLOs) can lease a selected part of the copper pair spectrum. For example, the OLO could lease non-voice spectrum, i.e., at frequencies above $4 \mathrm{KHz}$, which are used for DSL broadband access. In this case, the incumbent continues to provide voice (or other) services to its customers as the shared access loop is connected to its network, while the Digital Subscriber Line Access Multiplexer (DSLAM) is operated by the new entrant. The Custom Premises Equipment (CPE) is typically provided by the OLO. 
Other Licensed Operators (OLO) $\quad \square$ Local Loop Provider

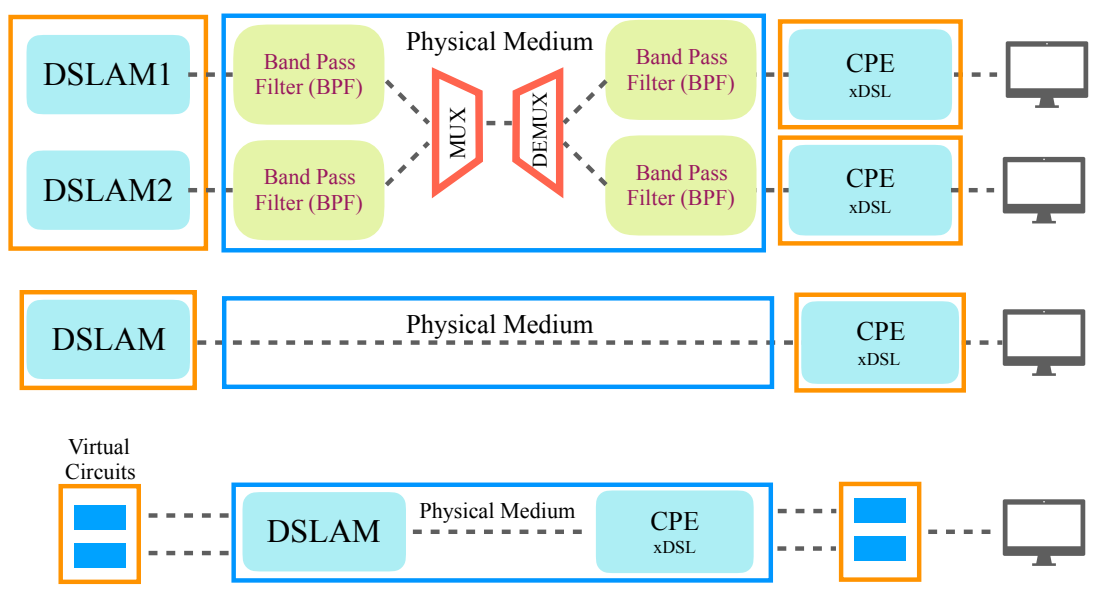

(c) Bitstream

Figure 1. Unbundling Techniques

One of the main challenges for line sharing was the hardware interface incompatibility. For example, the implementation of Asymmetric Digital Subscriber Line (ADSL) and Integrated Services Digital Network (ISDN) with telephony line required distinct spectrum allocations. Consequently, additional equipment was at times necessary for ADSL and splitting.

\subsubsection{Full Unbundling}

In the full unbundling architecture, copper pairs connecting the Main Distribution Frame (MDF) in the Central Office (CO) to the subscribers are leased to the OLO by the incumbent. When a customer requests to avail of its services, the OLO takes over the entire physical link from the MDF to the customer, as shown in Fig. 1b.

With full unbundling, OLOs have full flexibility in deciding physical layer parameters, including transmission technology, spectrum used, etc. As the incumbent leases the full physical medium, it cannot offer any service to that customer, until the line is released by the OLO (i.e., if the customer moves back to the incumbent operator) [4].

It is worth mentioning that when multiple operators deploy their access node equipment at the same location, their DSL service will experience crosstalk [5] which limits the achievable line rates. In principle, crosstalk cancellation (or vectoring) could help to increase the DSL networks' capacity significantly. While the Near-end Crosstalk (NEXT) can be avoided using standardized technology such as Frequency Division Duplex (FDD) and Time Division Duplex (TDD) [6], the Far-end Crosstalk Interference (FEXT) is the main performance-limiting factor. Spectrum management algorithms [7] and signal coordination techniques [8] can be used to mitigate FEXT Crosstalk. However, the formerly mentioned techniques are not easy to apply on brownfield DSL access since the current systems do not allow on-demand reconfiguration. In addition, if the copper pairs in a shared cable are handled by multiple operators, it is difficult to achieve crosstalk cancellation, which requires coordination across all copper pairs. In these cases, one solution for Crosstalk cancellation is correlating DSLAMs from different operators. For example, Cross-DSLAM Vectoring performs vectoring operations across multiple DSLAMs by coordinating them so that the vector group spans lines that terminate on different vectored DSLAMs [9]. However, this itself raises security problems because of shared management network [5]. In [10] the authors propose a sub-band vectoring solution for Multi-Operator environments which provides fair resource sharing between operators and meanwhile is quasi-optimal 
in terms of data-rate and does not impose the drawbacks of the Multi-Operator Vectoring architectures, such as deployment compatibility issues.

An alternative sharing technique is based on the concept of "Virtual Unbundling", like bitstream access and Virtual Unblunded Local Access (VULA), discussed in the next sections, which can reduce crosstalk as well as OLO's initial deployment costs [5].

\subsubsection{Legacy Bitstream Access}

Bitstream access was proposed in 2002 in order to enhance Internet access offers [11]. Bitstream provides OLOs with a new approach to utilize the incumbent's network through a new model that allows wholesale of DSL products.

The European Regulators Group (ERG) defines bitstream as "a high-speed access link to the customer premises with transmission capacity for broadband data in both directions [12]."

In bitstream, shown in Fig. 1c, the point of access for an OLO (traffic handover point) determines both the level of control over technical parameters and usage of OLO 's own network instead of the incumbents'. In principle, this provides the OLO with the choice on the amount of infrastructure to be deployed in the field, keeping in mind that this implies a trade-off with the level of control over the network performance.

As a consequence, the main drawback of bitstream is that OLOs can only offer a small number of different services (i.e., those defined by the incumbent). In addition, typically these are the same for all OLOs sharing the same infrastructure. This means that on the one hand, OLOs can only compete over price, as they cannot differentiate their service offerings among them. On the other, incumbents have little incentives to install new technology as there is no competition at the physical layer level with any of the OLOs.

\subsection{Next Generation Access (NGA) Bitstream}

NGA Bitstream is an enhancement of legacy bitstream, allowing operators to offer a wider range of high-speed broadband services without having to deploy their own access infrastructure. Figure 2 depicts the NGA Bitstream reference architecture. The supply chain of NGA Bitstream network architecture is made up of three main elements [13]:

- Delivery:

Traffic can be delivered to OLOs through various kinds of connections to the incumbent network. However, the delivery typically requires the use of a dedicated end device, usually owned by the OLO, which thus provides a level of independence from the incumbent's network. The decision of the delivery connection depends on the point of traffic collection for the OLO, which could be the closest incumbent nodes, a distant metro node or an IP node in the incumbent's backbone.

- Backhaul:

The backhaul is responsible for carrying the traffic from the local CO to the OLO 's network. This requires the traffic to be aggregated and classified into different classes of services (CoS). The two different models for traffic aggregation include a "shared bandwidth" model, where the backhaul capacity is shared between multiple access lines and the "dedicated model", where each line has a dedicated capacity. Depending on the choice of aggregation model, different service classification models can be used: a single CoS, where each VLAN only carries one traffic type and a multi CoS, where each VLAN can use multiple CoS values. 


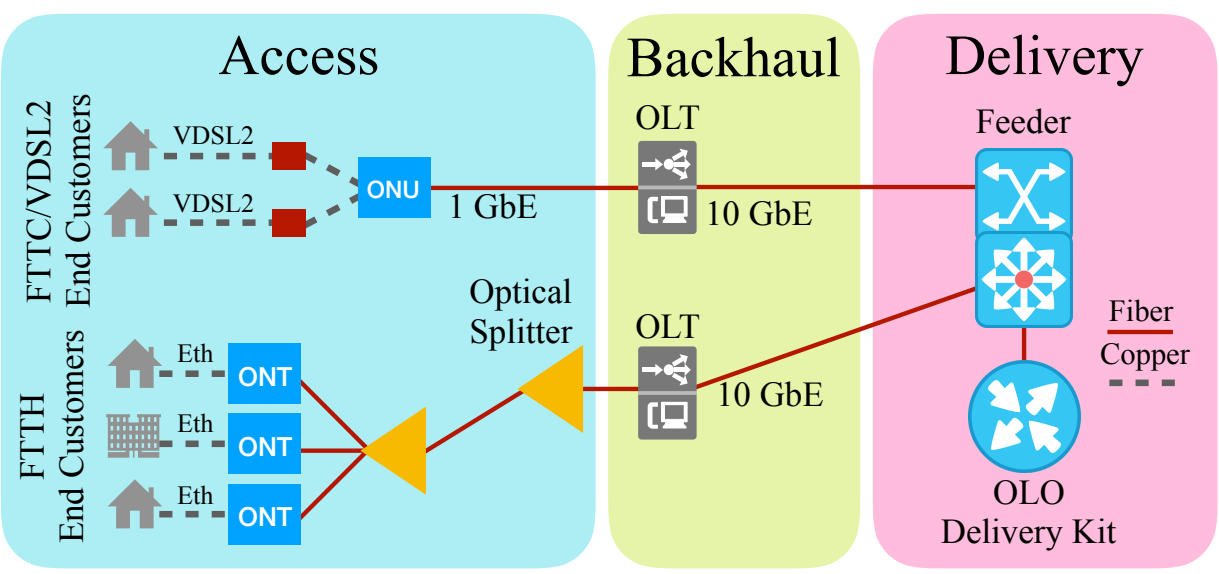

Figure 2. NGA Bitstream architecture

- Access:

The access network between the customer and the exchange point depends on the technology deployed on the customer side. If the technology used is fiber to the cabinet/VDSL2, the connectivity service will require a VDSL2 modem on the customer side. If the technology is fiber to the home, the optical network termination unit is required to be in the customers' premises. Other factors involved in the access portion are the device profiles (i.e., symmetric/asymmetric profile for upstream/downstream transmission) and the number of VLANs dedicated to each access line.

VULA is a regulatory tool that forms a compromise between the benefits of physical unbundling and the need to satisfy higher bandwidth targets [14]. Below we report the main differences with the NGA bitstream access mode.

- Delivery:

Unlike with NGA bitstream, in VULA traffic delivery can only occur at the exchange node level. This means that an OLO needs to collect its customers' traffic at any exchange where they have OLTs. There is no option to collect aggregated traffic at a central location. The incumbent operator installs a switch in each exchange node that is dedicated to VULA delivery, which will be shared among all OLOs requesting VULA delivery for that exchange node.

- Backhaul:

The main difference with NGA bitstream in the backhaul is that only the dedicated bandwidth multi CoS model is available for VULA. In addition, the backhaul bandwidth is not charged in the service.

- Access:

The access component of VULA is the same to NGA Bitstream, with the exception that the only type of user VLAN allowed is dedicated bandwidth multi CoS. VULA is quite similar to LLU from a functional point of view as it gives innovation and product differentiation.

\section{Sharing of Optical Access Networks}

Because fiber optic technology has large bandwidth capacity and low signal attenuation, it has largely superseded copper transmission in telecommunications networks. The only exception is in the network access, where short sections of copper lines are still used. Copper wire is used, in conjunction with fiber distribution, to deliver higher bandwidth DSL such as VDSL and G.FAST. Despite the cost of delivering new fiber to residential premises, an increasing number of operators are investing in FTTH deployment, in order to provide a future-proof mechanism to deliver higher capacity to residential and business clients. Indeed, optical access networks, in their different forms, are today the technology of 
choice for operators upgrading their network, offering high-speed broadband to both residential and enterprise users. Because optical access networks were deployed after the de-regulation of telecoms networks, line unbundling regulations do not necessarily apply, and different regions have adopted completely different approaches. The US, Europe and Japan are an example where different regulatory choices have led to different levels of FTTH deployment, with Europe and the US having experienced a much lower coverage than Japan [15].

Thus, sharing of optical access networks is today not common across the world. In places where sharing is in place, the approach has typically been limited either to fiber unbundling, that is, where the optical access is point-to-point fiber, or to higher layer NGA bitstream. In NG-PON2, where multiple wavelengths are available, it is possible, in principle, to separate OLOs by wavelength. However, there are still a number of technical issues. One issue relates to the ownership of the Optical Line Terminations (OLTs), as multiple wavelengths could interfere in certain cases, if not controlled by the same system. Another issue is that the allocation of a PON wavelength to one OLO is static and inefficient, preventing capacity unused by one PON to be used by other operators. Furthermore, NG-PON2 currently defines only 8 wavelengths for Time Division Multiplexing (TDM) access and the technology is not yet widespread, due to the high cost of the end-users tunable Optical Network Terminals (ONTs). More dynamic techniques have been discussed, for example in [16], [17], and could be made technically feasible with the recent development of SDN and NFV in the central office [18]. In the rest of this section, we briefly introduce some of the prevailing optical access technologies and review their major challenges with respect to multi-tenancy.

\subsection{Point to Point (PtP) Fiber}

The simplest optical network technology is the Point to Point (PtP) fiber, which provides a dedicated un-contended fiber path to each end-user towards the central office.

PtP optical networks are most suitable when it comes to transferring high volumes of data for specific purposes such as enterprise access or FTTE (fiber-to-the-telecom-enclosure). In many cases, the PtP optical link carries data for residential users up to a switching point, where traffic is then distributed across a number of other technologies (e.g., Ethernet and WiFi). However, in a small number of cases, it is also used as a residential access technology. PtP has the main disadvantage of requiring one fiber strand and one active port in the $\mathrm{CO}$ for each end-user, leading to increase in cost, energy consumption and footprint. These shortcomings have led to rethinking this architecture and considering other options in the last mile.

PtP fiber provides a simple method for network sharing, as it can enable full physical unbundling. Different operators can simply patch their customers' network termination point into their own equipment.

\subsection{Time Division Multiplexing Passive Optical Network (TDM-PON)}

TDM-based PONs are a series of fiber-based broadband access network technologies that offer numerous advantages when deployed in fiber to the home (FTTH) scenarios. They are based on point-to-multipoint tree topology, using optical power splitters, where one OLT at the network side can serve several end-user ONTs. Downstream, the PON operates as a broadcast and select architecture, making bandwidth management relatively simple. Indeed quality of service can be implemented following typical Layer 2 or Layer 3 queue management tools. On the other hand, a more complex bandwidth allocation mechanism, called Dynamic Bandwidth Allocation (DBA), is required in the upstream direction in order to avoid collisions among the multiple ONTs transmitting towards the OLT.

The number of end-users of a PON can vary substantially. In today's commercial systems it is typical to have 32 or 64 endpoints, although the protocol can support more, and architectures have been presented, justifying split ratios up to 1024 [19]. The available capacity in commercial PONs is of the order of 10 Gbps symmetric rates per channel, with up to 8 channels in NG-PON2. Current 
standardization efforts are finalizing $25 \mathrm{~Gb} / \mathrm{s}$ per channel, while future release will aim at 50 and 100 $\mathrm{Gb} / \mathrm{s}$. Such high capacity is an enabler for PON infrastructure sharing.

In addition, the capillarity of fiber access points that PONs enable, once it's deployed for residential access, have spurred interest from mobile operators to consider it as backhaul or fronthaul solution for their base stations. This will further diversify the network requirements and will make the shared PON's Quality of Service (QoS) more complex. The adoption of PONs for backhaul [20], fronthaul [21] in cellular networks and 5G networks [22] have recently been investigated by many researchers.

In [23] the authors proposed an inter-operator fixed-mobile network sharing approach, where the operators can divert their access-network traffic to other operators, using inter-operator communication, to achieve higher performance or improve availability. Packet tagging is then used to divert the traffic back to the original operator. This work, however, does not account for the PON DBA, described below. When sharing the PON across multiple operators (potentially offering different services), a reliable quality of service scheme is required to guarantee sustainable service delivery to the end-users. In other words, the sharing shall not prevent the operators from implementing their QoS scheme (including the DBA) to meet diverse service-dependent requirements. Such a heterogeneous scenario requires an ultra-flexible and customizable control model to provide tenants with adequate control over the resources leased from the infrastructure provider [24]. Thus, new radical thinking is needed to enable heterogeneous PON sharing.

One of the essential control features of a PON is the DBA algorithm, which provides TDM scheduling to the ONTs for upstream transmission. DBA is responsible for collision prevention, utilization of the upstream bandwidth and providing the required QoS to meet Service Level Agreement (SLA) requirements. Therefore DBA is one of the essential parts of the PON control plane that can satisfy the requirements of the users. However, in a heterogeneous scenario, performance metrics such as latency and QoS can be conflicting goals. The well-known trade-off in DBA is the QoS, fairness and bandwidth allocation accuracy versus latency, i.e. the DBA algorithms with higher accuracy in reporting the queue occupancy (and as a result better QoS, fairness and bandwidth efficiency) can impose higher latency on the PON scheduling.

Cloud Radio Access Networks (C-RAN), for example, pose a very stringent latency threshold of around 150 microseconds [25] for the Remote Unit (RU) to Distributed Unit (DU) optical link. This is only one of the many applications requiring low latency. While for example the concept of inter-vehicle communications has been around for almost two decades [26],[27], today, following the development of autonomous vehicles, support for Vehicle-to-Vehicle (V2V) [28] and Vehicle-to-Anything (V2X) [29] communication is becoming a real requirement for $5 \mathrm{G}$ networks. Since V2X requires ultra-low latency at the application level, this will pose low latency backhaul requirements even when the mobile cells serving the V2X ecosystem operate over a Distributed RAN or a C-RAN with a high layer functional split.

The conventional DBAs in TDM-PONs have a latency of the order of several hundred microseconds to milliseconds [30]. Most of this latency is due to the need for the OLT to receive Dynamic Bandwidth Report upstream (DBRu) in order to assess the ONT capacity requirement. Therefore, DBA mechanisms capable of providing ultra-low latency are desired. Some examples of such low latency DBAs have been proposed in [31-33] mainly based on cooperation with the Long Term Evolution (LTE) scheduler to map the wireless resource blocks to PON bandwidth maps and achieve low-latency.

On the other hand, other service providers might have completely different requirements, leading to a different trade-off between latency and bandwidth efficiency. Thus, they would require to operate different versions of DBA to meet their requirements. Such customization is, however not possible in current OLTs' control planes, and all the tenants are compelled to settle for the DBA implemented by the Infrastructure Provider/Vendor. 
An intuitive solution for PON multi-tenancy would be to use multiple OLTs over the same optical distribution network (ODN). In [34] the authors propose upstream and downstream DBA algorithms for such multi-OLT PONs. However, dedicating an OLT for each PON tenant will impose more cost to the Infrastructure Provider (InP), resulting in the increase of CapEx and OpEx and inefficient use of capacity, in addition to increase in overall energy consumption. It is worth mentioning that the popularity of PONs in access networks relies on its passive nature, therefore, increasing the ratio of active to passive elements will result in a potential decline in PON popularity. Thus, the ability to multiplex multiple tenants on each OLT is a highly desirable feature.

Chengjun et al. $[35,36]$ have proposed a slice scheduling scheme capable of assigning bandwidth slices to different tenants. Each slice of a PON bandwidth resource is defined as an upstream XG-PON frame. The Slice Scheduler is an interface located between the network operators and the PON transmission convergence layer to enable several operators to control their share of bandwidth resources in a TDM manner. The second stage is the frame scheduling stage, which lets each operator employ their customized DBA to serve their ONTs. As mentioned by the authors, there is a trade-off between the isolation and efficiency of bandwidth allocation. A completely isolated scheduling referred to as static bandwidth resource sharing (static BRS) - would waste the excess bandwidth of lightly loaded operators. The authors solve the issue by proposing also a dynamic BRS. This achieves achieves better PON utilization but at the expense of less isolation between the tenants (e.g., in terms of latency dependence). In addition, separating operators by assigning different frames increases the latency seen by each operator, which also becomes dependent on the number of operators sharing the PON.

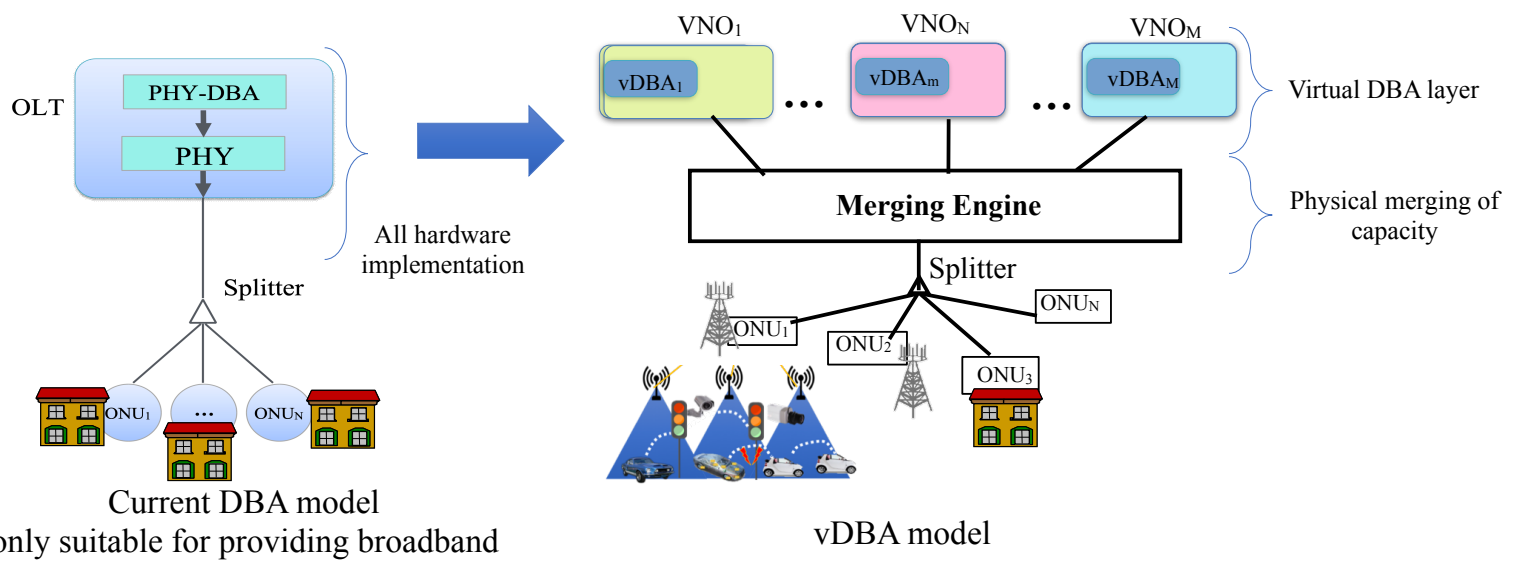

Figure 3. DBA Virtualization

The concept of DBA virtualization was introduced in [37]. Fig. 3, shows its principle (drawn on the right-hand side), compared to the default physical DBA (left-hand side). The objective of virtual DBAs is to allow VNOs to implement their own version of DBA, independently from the infrastructure provider. The procedure of the bandwidth allocation starts with the Merging Engine forwarding incoming buffer occupancy messages from the ONTs to each virtual DBA (vDBA). Next, each vDBA issues a (virtual) bandwidth map for their own ONTs, using customized DBA algorithms to fulfill their requirements. All virtual bandwidth maps are then collected by the Merging Engine, which merges them into a single physical bandwidth map, which is broadcast to all ONTs. The reported results show that this approach can enable true multi-tenancy, while not imposing any additional delay to the PON scheduling, thus achieving adequate isolation across operators.

The study of PON sharing is in its early stages, and more research is currently being carried out on this topic. The recent progress in NFV architectures and frameworks represent an excellent opportunity to boost the virtualisation and slicing ability for PONs, which are essential for improving isolation. Along with NFV, SDN can provide a suitable framework for the orchestration of the virtualized functions of the PON. 


\subsection{Wavelength division multiplexing PON (WDM-PON)}

Another approach to PON is to split the distribution network in wavelengths rather than in power. This requires the use of wavelength demultiplexers in the Optical Distribution Network (ODN) in place of optical splitters, so that each end-user can be connected to the $\mathrm{CO}$ through a dedicated wavelength. In this case, the fiber network remains as a point-to-multipoint structure, although the connectivity is provided as a point-to-point wavelength channel overlay. This architecture goes under the name of WDM-PON. The main advantages are that it provides isolation between channels and does not require upstream bandwidth scheduling algorithms. This second point is an advantage for providing services such as fronthauling of Cloud-RAN, as it provides low and deterministic latency $[38,39]$. In addition, sharing can be achieved by allocating wavelength channels to different operators, according to their customer connection. Nonetheless, this isolation comes at the cost of dedicating a wavelength and a termination port at the central office to a specific end-user, and it requires every end device to operate tunable lasers. In addition, static allocation of wavelengths to end-users is inefficient, since similar to PtP fiber, any unused capacity cannot be assigned to other users.

In the past, however, researchers have proposed solutions to tackle such inefficiency. For example, in [40], a purpose-built WDM-PON is introduced in which service providers can transport residential and business traffic as two virtual networks on one physical PON.

In [41] three different unbundling strategies for realizing multi-operator GPON were compared, with respect to ease of market entrance for new network operators, based on their deployment costs. The first approach is moving back the PON splitter into the $\mathrm{CO}$ to mimic an architecture similar to a P2P in which a single fiber is dedicated to each customer. The second strategy is replicating the access network, i.e. replicating the fiber deployment in the drop segment by dedicating a splitter to each operator. The last option is upgrading an already-deployed TDM PON to WDM PON. The cost analysis was done for scenarios with a low to high density of customers per square kilometer, and the conclusion was that upgrading to WDM is the most efficient strategy to perform LLU using GPON. Indeed, if we assume the constraint that the sharing needs to be achieved through channel unbundling, WDM-PON can be seen as a natural solution for multi-tenant PONs due to the ease of sharing it provides. Each VNO can operate on one of the wavelengths and serve its users with absolute isolation from the other VNOs. However, the same limitations of PtP fiber discussed above apply, including additional cost, footprint, energy consumption and inefficiency of capacity allocation.

\subsection{TWDM-PON}

Time and Wavelength Division Multiplexed Passive Optical Network (TWDM-PON) was designed to increase the TDM-PON capacity by providing multiple wavelengths over a power-split architecture. This was standardized in 2012 by FSAN as the Next-Generation PON Stage 2 (NG-PON2) architecture [42]. The main advantage of this architecture is that it increases the overall PON capacity while maintaining the flexibility of a TDM-PON, as end-users can, in principle, be moved across wavelength channels for load balancing. In addition, this technology opens up a number of additional possibilities for network sharing, as discussed in [16]. For example, OLOs could be assigned a wavelength, to which the end-users' ONTs can tune according to their preference. Or the ONTs can be assigned dynamically to different OLTs for online load balancing purpose, while the multi-tenancy is addressed at the capacity scheduling level.

The flexibility provided by TWDM-PON has spurred a number of novel ideas for network sharing.

In [43], the authors have proposed a Multi-OLT and multi-wavelength optical access network which enables each ONT to be shared among all operators. However, their solution does not support simultaneous time and wavelength allocation, and in the upstream, each service operator uses an individual wavelength. Therefore it causes a channel separation among the operators, which leads to low utilization of the channel. Since operators might have different data rates and packet lengths, the advantage of the multi-OLT architecture proposed in [43] relies on reducing the burden of data processing and management compared to the case of using a single OLT for all operators. 
In [44], the authors propose a dynamic TWDM-PON to bring flexibility to mobile Radio Access Network (RAN). The proposed technology enables a virtual PON to be configured with a wavelength so that it can be effectively applied to a RAN scenario. The virtual PON dynamically responds to the changes in the capacity requirement, which improves bandwidth efficiency. Their numerical simulations verify that the proposed dynamic TWDM-PON can accommodate twice as many user terminals as a fixed PON.

\section{Software Defined Network (SDN)}

Initially devised as a mechanism to separate control and data plane in network switches and routers, SDN has in only a few years evolved into a comprehensive framework to add flexibility and programmability to the entire telecommunications network. These features have led to SDN being considered as an essential element to deliver network multi-tenancy.

\subsection{Multi-Tenancy in SDN Controllers}

The first attempt to enable some level of multi-tenancy on an OpenFlow-based control plane was the FlowVisor implementation [45]. FlowVisor is a transparent Proxy Controller that slices resources and provides data forwarding programmability to the tenants. FlowVisor, however, did not enable network topology abstractions, e.g., for path splitting and path migration functions [46]. Thus, it only partially supported network virtualization.

Argyropoulos et al. [47] have proposed and analyzed three control plane slicing methods (switch, port-wide and domain) implemented by the management plane, which safeguards control plane isolation among tenant virtual networks. They have introduced a Flowspace Slicing Policy (FSP) rule engine, which is an automated mechanism for translating substrate management plane policies to virtual network mapping control plane rules. Their work complements that of the FlowN [48] technique by introducing slicing methods that are sufficiently generic to permit enforcement of n!

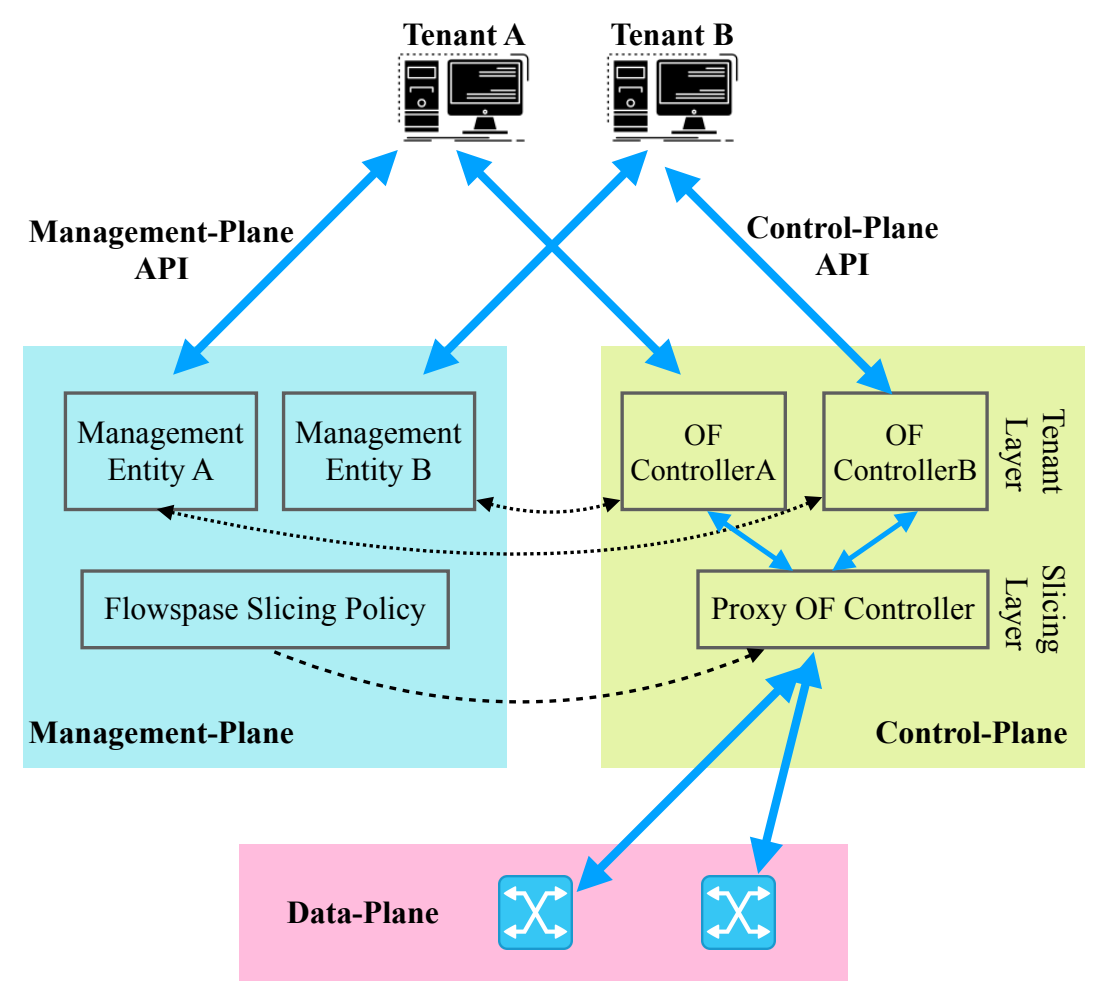

Figure 4. Proxy Controller-based architecture 


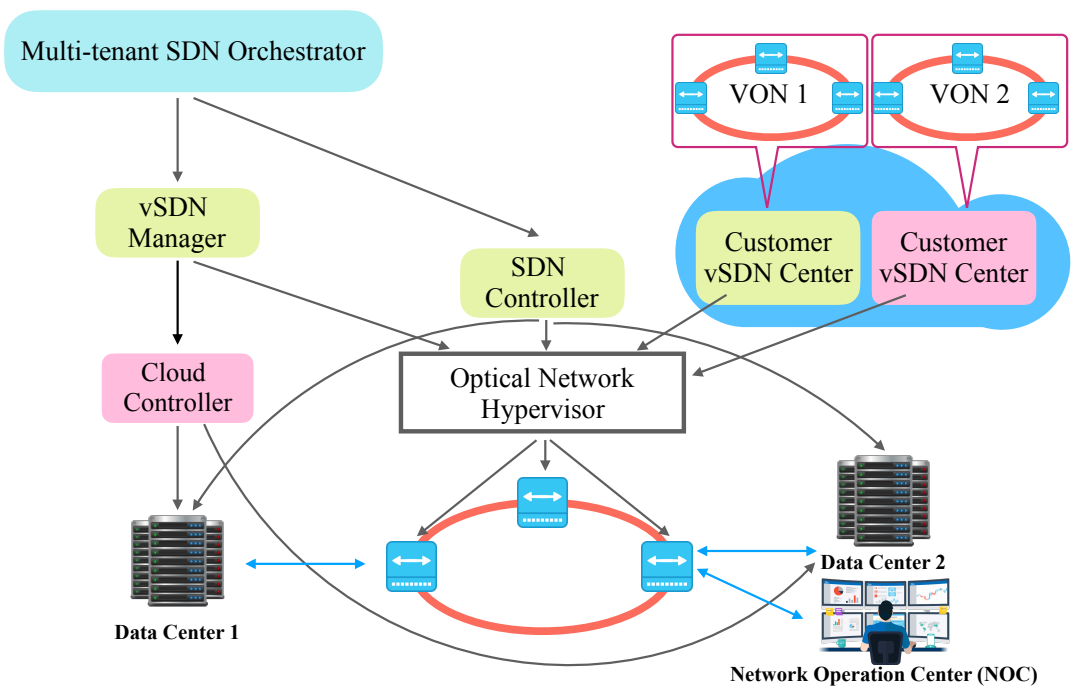

Figure 5. SDN/NFV Orchestration Architecture for Multi-tenant Optical Transport Networks with Virtual SDN Controllers [50]

Figure 4 shows that Flowspace delegation in SDN can be executed using an intermediate control plane slicing layer. The Flowspace slicing policy is designed according to the control plane slicing method, which is an algorithm that guarantees the creation of non-overlapping Flowspace rules. A Proxy controller (e.g., FlowVisor) routes control messages transparently. The other option can be based on the network hypervisor. In this architecture, the control plane consists of a slicing layer, a virtualization layer and a tenant layer. The virtualization layer incorporates virtual network mapping algorithms. In contrast, in the proxy controller architecture, it is the tenant layer of the hypervisor that co-hosts the forwarding logic of the tenants.

One of the practical problems in multi-tenant switching architectures is that of packet classification. Shared infrastructure controllers typically use L2 or L3 header fields as slice identifiers. However, using packet IDs to classify data-plane Flowspace slices can fail if the number of slices is above the range of packet ID values. To tackle this scalability problem, Argyropoulos et al. [47] have considered logical separators that are also linked to data-plane resources. They have experimented with the domain, switch and port-wide slicing techniques. Their experiments focused only on two architectural approaches for control plane virtualization in OpenFlow enabled multi-tenant SDN domains. Their experiments demonstrate that port-wide slicing technique is most efficient in terms of tenant request acceptance ratio within acceptable memory consumption and control-plane delays.

Another approach targeting scalability in hypervisor design is that presented by Bozakov et al. [49], introducing a virtualization layer that automates the deployment and operation of SDN slices on top of a shared network infrastructure. They have named their technique as AutoSlice (Automated and Scalable Slicing for SDN). The proposed hypervisor consists of the management module and multiple controller proxies. Scalability is ensured through the development of auxiliary software datapaths (ASD) in the substrate network. The physical substrate is segmented into multiple SDN domains and has assigned a dedicated controller proxy. Each controller proxy transparently manages access to corresponding domain switches.

The work from Munoz et al. [50] has further extended the concept of network and control plane virtualisation, by moving the SDN controller to the cloud for dynamically deploying independent SDN controller instances. This process takes a few minutes to deploy a new virtual optical network instance. This represents a considerable advantage compared to the several days that it would otherwise take to manually install and configure an SDN controller on a dedicated server. In addition, moving the controller to the cloud has other obvious advantages of removing hardware maintenance downtime and decreasing recovery time in case of failure. 
In their proposed architecture, reported in Fig. 5 the NFV infrastructure is comprised of an optical transport network interconnecting distributed data-centres, providing storage, compute and network hardware resources. On top of the physical infrastructure, they have deployed the NFV virtualization layer, which virtualizes storage, compute and network resources of NFV infrastructure. Above the virtualization layer, they have deployed various Virtual Network Functions (VNF) managers to manage the VNF life-cycle. They have proposed a VNF manager, called vSDN controller manager, which requests the cloud controller the creation of VMs and installation of OS images with compiled Floodlight or OpenDayLight SDN controllers. Finally, on the top, there is the orchestrator for multi-tenant $\mathrm{SDN}$-enabled transport networks.

This work was further extended in [51], where the authors combined NFV and optical network virtualization, implementing an on-demand OpenFlow-controlled virtual optical networks (VON). Every tenant SDN controller runs on the cloud to control the deployed VON. The users of virtual optical networks can create, modify and delete virtual network slices dynamically in response to application demands. The users' SDN controllers can control the allocated virtual network resources independently. This is achieved by the Optical Network Hypervisor $(\mathrm{ONH})$ which slices the transport network into multiple virtual optical networks and represents an abstract topology of each virtual optical network to corresponding users' SDN controllers. Moreover, the SDN controller allows controlling virtual optical networks remotely through well-defined interfaces. Their implementation was deployed in the ADRENALINE testbed, practically demonstrating how the NFV orchestrator can provide multi-tenancy on top of the heterogeneous transport networks.

\subsection{Software Defined Optical Access Networks}

Considering that the access network is the part where multi-tenancy has its biggest value and also its major challenges (as mentioned throughout section 2 of this paper), it is natural to apply the advantages brought by NFV and SDN to the access and to PONs in particular.

Initial access virtualisation concepts were introduced in [52], with a Software Defined Access Network (SDAN), where access network management and control functions are virtualized. This can speed up service creation, streamline operations and enhance customer satisfaction in multi-operator environments. SDAN works with port-level, physical cable and logical bitstream unbundling. SDAN moves storage and computing functions from Network Elements (NEs) to the controller and provides a common interface to control functions accessed by multiple operators.

Around the same time, architectures started to appear for applying SDN to PONs. For example, Clegg et al. [53] have described an architecture enabling OpenFlow on multiple access technologies (point to multi-point devices) with minimal changes. Their concept is shown in Figure 6, depicting the architecture of an OpenFlow enabled Ethernet Passive Optical Network (EPON).

The main feature in this architecture is the HAL (Hardware Abstraction Layer) box, which links to the OLT management port. Blue lines represent Ethernet frames and dashed green lines are the optical section of the device. The proprietary control path to the management interface is represented by a black piped line, whereas the dotted red line represents OpenFlow control messages. The major role of HAL is to map port pairs/VLAN on the physical system with virtual ports on virtual distributed OpenFlow switch. 


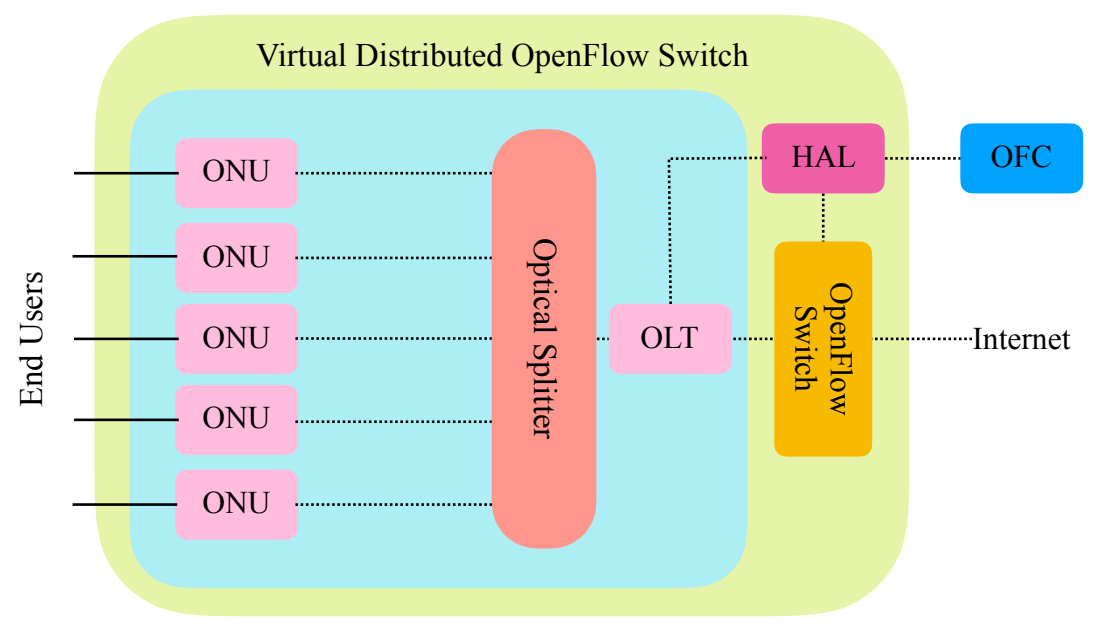

Figure 6. The architecture which allows the EPON to use OpenFlow

The next obvious step was the virtualisation of the entire $\mathrm{CO}$, which was brought about by the Central Office Re-Architected as a Data Center (CORD) [18]. This project proposed to provide a new telco central office architecture aiming to replace the large amount of proprietary purpose-built hardware components with software running on commodity servers and off the shelf white box switches and access devices. Therefore, implementing the central office as a data center rather than a traditional architecture, which often required up to 300 unique hardware devices with a broad range of technology and requiring large CapEx and OpEx to operate. CORD uses XOS [54], a service orchestration layer built on top of OpenStack [55] and the ONOS SDN controller. The project, originally funded by the ON.LAB, is now under the umbrella of the Optical Network Foundation (ONF), which counts a considerable number of collaborators and partners, including some major service providers and network equipment vendors. The CORD architecture creates a suitable environment for realizing the centralization approaches such as C-RAN. The Project consists of three sub-projects, namely residential CORD (R-CORD), Mobile CORD (M-CORD) and Enterprise CORD (E-CORD). Each sub-project is a proof of concept use case for the CORD framework for demonstrating its ability to accommodate a wide range of technologies in a software-defined architecture.

$\mathrm{R}-\mathrm{CORD}$ is the sub-project that focuses on the last mile access networks for the residential market. It's based on the use of PONs as its infrastructure and implements the SDN idea by virtualizing all network components, from the OLT to the ONT, in addition to the other CO elements common to the more general CORD implementation. The advantages of such new architectural concept are manifold, from reduction of CapEx and OpEx (e.g., due to commoditisation of CO infrastructure and ability for multi-tenancy) to increased revenue, generated by unprecedented flexibility that can support new and more dynamic services, on-demand.

For instance, ONTs and OLTs are now programmable units, which can in principle allow on-demand adjustment of overarching rules and policies to match incoming traffic flows to logical connections. This can be applied as required to suit diverse and new services.

One specific example of such flexibility and programmability is its application to the Dynamic Bandwidth Allocation algorithms in the PON. From early approaches based on creating isolated groups of PON users [56], to complete frameworks for the virtualisation of the DBA (vDBA), previously introduced in section 3.2. This extends the OLT virtualisation down to the data plane [57], by also virtualising the OLT scheduler [58]. The result is the ability for different VNOs to operate different DBA scheduling algorithms, so that they can fine tune the scheduling to suit specific business models, or even applications, running in their PON slice. The concepts were also showcased at two public testbed demonstrations [59],[60]. 
Similar ideas were also proposed for QoS handling in the downstream direction [61], where a novel scheduler was designed to handle QoS differentiation simultaneously at the service, customer (i.e., ONT), OLT and VNO level [62].

\section{Fixed Access Network Sharing Economics}

We have already explored the principles of fixed access network sharing and the technologies that enable it. We have seen that, for example, multi-wavelength systems, such as NG-PON2 can provide both high capacity, isolation and flexibility of operation in shared networks. However, one of its main disadvantages is that it requires ONTs to be equipped with tunable lasers and filters, making them expensive. Indeed, the body of European Regulators for Electronic Communications (BEREC) has published a Report [63] on the New Forms of Sharing Passive Optical Networks Based on Wavelength Division Multiplexing. In a BEREC questionnaire completed by 50 European network operators, more than 20 per cent of the operators have mentioned that the expense of the NG-PON2 equipment was one of the main reasons why it is not likely that the network operators will deploy NG-PON2. On the other hand, only four operators have considered wholesale wavelength unbundled services and the reuse of the passive network infrastructure as primary reasons for the network operators to deploy NG-PON2 [63]. We have seen 3 that Time Division Multiplexing is an enabler for network sharing. However, network operators are unlikely to invest in fine-grained sharing models, without some economic incentives.

In this section, we look at some of the economic incentives for Fixed Access Network Sharing (FANS) that involve new ownership models.

\subsection{Fixed Access Network Sharing for $5 G$}

Considering cooperation and competition amongst operators, in [64] the authors study how the Swedish telecommunications business landscape changed throughout the different mobile network generations (GSM, 3G, and 4G) and competing mobile operators started to share network resources. However, this trend changed with the deployment of $4 \mathrm{G}$ networks, where reduced equipment costs and re-usability of the base station sites between $3 \mathrm{G}$ and $4 \mathrm{G}$ played a role in disincentivizing operators to share. Based on the market reports in [65], the upgrade pattern to $5 \mathrm{G}$ will be radically different from 3G and 4G, where an increment of 23\% in CapEx is expected between 2018 and 2025.

In [66] the authors conduct a cost assessment studying how PON/FTTH network could affect factors such as initial investment, cost per home connected and the payback period. Their study covers the most popular optical access technologies and standards, namely GPON, XG-PON, TWDM-PON, and AWG-based WDM-PON in urban and suburban regions. They conclude that while employing a network-sharing scheme increases the cost per home connected and the payback period, the required initial investment is strongly reduced.

These solutions bring cost savings of up to $40 \%$ in terms of CapEx, and up to $15 \%$ in OpEx over a five-year period [67]. By other figures, the number of announced network sharing agreements between network operators worldwide has increased almost 20 fold in 7 years (five agreements in 2010 and 98 in 2017) [68]. We believe this vision will require the operators to think beyond simply sharing the feeder fibre cables or site-reduction [69] as a cost-saving approach towards their infrastructure.

In addition to cost reduction, infrastructure sharing can facilitate the expansion of coverage, therefore, helping the operators to grow their customer base and access new sources of revenue.

The large potential capacity of optical access networks makes a strong case for the operators to reduce their costs by exploiting the efficiency of scale that can be achieved by sharing. This is especially important to facilitate new entrants and promote competition. However, this comes at the cost of incentivizing operators to share their infrastructure and resources. In some countries, the regulator may attempt to enforce sharing, but this has been met with limited success as operators try to circumvent regulations by using legal loopholes, for instance, by not providing the required interfaces. We believe that as the cost for $5 \mathrm{G}$ network deployment soars, the potential reduction in the total cost 
of ownership (TCO) achievable through new models of infrastructure sharing will provide a better driving force than the legacy regulatory enforcement.

SDN and virtualization can facilitate sharing primarily in two ways :

1. By Providing simplified and standardized interfaces to connect to other operators network.

2. By virtualizing the critical network control functions and provide customizable functions for the guest operators.

\subsection{New Ownership Models}

As 5G networks promise unprecedented support for novel heterogeneous services, new business and ownership models are required that take into consideration their entire value chain, that is, InP, network operators and over the top service providers. To achieve the target sharing level, all parties will be required to collaborate and cooperate regardless of the potential competition among them. This is not an easy goal to achieve as they often have conflicting interests, which could be a serious obstacle to their commitment. Thus, a robust mechanism to assure the commitment of all the parties is required. The study of interactions among parties with conflicting interest is not a new field, neither in economics nor in telecommunication networks. Indeed, the application of game theory to economics is solely dedicated to resolving such situations, where the strategic interaction between decision-makers are involved in a collective decision-making process. These parties might have conflicting and contradicting interests; therefore, they are more committed to achieving a better outcome for themselves than for the system as a whole.

Game theory has been widely used to solve collaborative resource sharing problems in a wide range of subjects, including computer science, telecommunications, management, etc. One of the most successful examples of game theory applied to resource sharing is the wireless spectrum sharing in telecommunication networks. Initially, it was used in primary spectrum licensing, which involves one time nationwide auctioning of the scarce spectrum, usually conducted by the governments. However, such long-term fixed spectrum licences (e.g. latest ComReg's 3.6 GHz Band Spectrum award for the duration of 15 years [70]) to primary users leads to low utilization of the spectrum (more than $70 \%$ of the radio spectrum, in certain times or geographic locations [71]). The inefficient use of spectrum has prompted the regulators to investigate the secondary use of the licensed spectrum, where the primary users can improve the utilization of the spectrum allocated to them by enabling the reuse of the underutilized bands by secondary users [72].

The fixed access sharing is very similar to the spectrum licensing, as for instance, current sharing methods of dedicating entire fibre or wavelength channels lead to low utilization of the access network capacity. Thus, an opportunistic secondary sharing scheme could be adopted to assure higher utilization of the network. Considering the reusable capacity of the FANS as a tradeable commodity and a market-based sharing scheme for the under-utilized capacity sharing, the following issues need to be addressed:

1. Lack of trading activity [73]: Operators' unwillingness to join and participate in the market, which could lead to limited tradeable resources, therefore, lack of sufficient liquidity in the market.

State-of-the-Art solutions:

- Providing sufficient control over the critical network functions, such as scheduling [37], so that operators can offer QoS-oriented services to their customers (see section 3).

- Providing participation incentive through monetary compensation. In [74],[75] a marketplace is proposed to allow multiple network operators to utilize a passive optical network infrastructure and reuse others' under-utilized capacity. This marketplace provides monetary compensation to the operators who share their excess transmission opportunities. The market assumes an ownership model where an infrastructure provider owns the entire 
PON and allocates a certain capacity to the virtual network operators, which can trade their excess capacity among them. The network operators will benefit from this as they can monetize their idle resources and in peak usage times serve their customers with a higher Peak Information Rate (PIR). The InP also enjoys some advantages as it can utilize its resources more efficiently. Finally, the concept of purchasing assured capacity-on-demand at small granularity can support novel, revenue-generating applications, which require deterministic delivery of network capacity to operate correctly (e.g., those based on augmented reality).

2. Anti-competitive behaviour, including hoarding of resources and excessive pricing [73].

State-of-the-Art solution: Economically robust auction mechanisms designed for preventing manipulative market behaviours. For example, in [76] an auction mechanism is proposed for a shared PON, which provides positive incentives for the operators to avoid malicious conduct in the market. The proposed double auction can support simultaneous multiple-item trades. It does not impose any additional communication delay to the time-critical scheduling process of the PON as it relies on a sealed-bid bidding process which, unlike common open auctions, does not involve any tug of war bidding among the participants.

3. Lack of trustworthy central authority, including scenarios where the infrastructure provider is also a competing operator.

State-of-the-Art solution:

Most of the scenarios in the state-of-the-art have considered cases where infrastructure is provided by a trusted third-party (e.g., a government authority). The InP is assumed to be trusted by all of the parties and provides a secure and reliable platform for the networks operators to trade. However, this approach overlooks the other network ownership models where either the InP is not trusted by all of the participants to be entirely impartial, or an ownership model does not involve a central $\mathrm{InP}$, and the role of providing the infrastructure is distributed among the operators. Blockchain technology uses a distributed consensus mechanism relying on a distributed ledger to assure trust among a number of participants without a central entity. Empowered by smart contracts (i.e., a piece of code that digitally verifies and enforces a contract), Blockchain can take a step forward and operate trustworthy technical/business processes with no intermediary involved. In [77], the authors describe how smart contracts can facilitate the automation of complex multi-step processes in an Internet of Things (IoT) ecosystem. The application of Blockchain in the creation of machine to machine service and resource marketplaces has also been addressed in [77]. In [78], the authors have studied a blockchain solution for network slice brokering in 5G networks. In [79] a blockchain-based distributed bilateral trade mechanism is introduced. Using the mechanism presented in [79], bilateral trade markets that are widely used in telecommunication networks (e.g., resource allocation in NFV markets [80], femtocell access [81], mobile crowd sensing [82], spectrum sharing [83] and PON Sharing [76]) can function in an untrusted environment.

The proposed solutions facilitate leasing resources from infrastructure providers dynamically according to the needs of the operators. The same approach could also come into help in FANS peer-to-peer trust issue. However, further research is required to address application-specific requirements in FANS, such as latency limitations.

These findings open up new possible research areas. For example, in [84] a CapEx cost model for PON technology has been proposed. However, this cost model does not account for infrastructure sharing. A greater focus on designing a cost model for fixed access network sharing could produce interesting findings when also considering more heterogeneous use cases, e.g., merging residential, business and Cloud-RAN services. In [85], for example, the authors investigate the advantage of PON sharing for implementing distributed Multiple Input Multiple Output (MIMO) in C-RAN. Additional 
work could provide a quantitative analysis of the OpEx reduction enabled by the deployment of Blockchain and smart contract technology. This can significantly reduce human intervention in the business transaction part of the service chain, thus reducing service provisioning time and increasing the network revenue.

\section{Summary and conclusions}

This paper has highlighted the critical role that sharing of optical networks will play in the evolution of future networks, beginning with 5G. We reviewed the largely regulatory driven models that are currently used for access networks sharing and we highlighted their major challenges. We see that the major shortcoming of current sharing models is their inability to support diverse requirements when the network is utilized by multiple operators that want to provide a wide range of services to their customers. This limitation is a bottleneck for $5 \mathrm{G}$ networks, one of whose main novelty is their ability to support highly heterogeneous applications. We conducted a case study of more recent multi-service PON sharing and highlighted current challenges and limitations and tentative state-of-the-art solutions that overcome these limitations. Our findings suggest that functional virtualization, along with SDN, could play a vital role in overcoming the challenges, since these technologies enable flexible management for operators, which is especially needed in the network access.

In section 5, we speculated on how the $5 \mathrm{G}$ evolution might affect the current network ownership models, challenging the conventional roles of InP and Virtual Network Operators (VNOs) and demanding new ownership models. We investigated some of the challenges for network operators sharing a common PON infrastructure and discussed the possibility of new ownership models.

We briefly introduced the state-of-the-art solutions to some of these challenges, including a market model to provide trading incentives through monetization of the excess resources and then modelling the multi-tenant PON as a bilateral trade market. Multi-tenancy could potentially facilitate new partnership and co-investment models for network operators. In this report, we addressed one such model in which a trusted infrastructure provider is the sole provider of the resources. However, more complex sharing models are yet to be addressed as new network ownership/operation models emerge thanks to the network virtualization.

Finally, we conclude this paper with a few insightful remarks on possible future research:

- Designing and regulating new access network ownership models should be a key policy priority to ensure smooth deployment of 5G networks.

- More research is needed to determine the business implications of the new ownership models, presumably utilizing novel approaches such as blockchain and smart contracts to assure economic robustness and trust.

- Further studies should assess the potential for other network component/function virtualization opportunities to enhance the flexibility of the shared access.

Funding: Financial support from Science Foundation Ireland grants 14/IA/252 (O'SHARE) and 13/RC/2077 is gratefully acknowledged.

\section{References}

1. Meddour, D.E.; Rasheed, T.; Gourhant, Y. On the role of infrastructure sharing for mobile network operators in emerging markets. Computer Networks 2011, 55, 1576 - 1591. Recent Advances in Network Convergence, doi:https:/ /doi.org/10.1016/j.comnet.2011.01.023.

2. 5G-PPP. The 5G Infrastructure Public Private Partnership: the next generation of communication networks and services., 2015.

3. Bauer, J.M. Local loop unbundling and bitstream access: regulatory practice in Europe and the US; DIW Berlin, 2006.

4. Gabelmann, A. Regulating European telecommunications markets: unbundled access to the local loop outside urban areas. Telecommunications Policy 2001, 25. 
5. AlcatelLucent. Dynamic Line Management for Vectoring Scenarios.

6. Fazlollahi, A.H.; Wang, X.; Zeng, Y. FEXT Exploitation in Next Generation DSL Systems. 2016 IEEE Global Communications Conference (GLOBECOM), 2016, pp. 1-6. doi:10.1109/GLOCOM.2016.7841840.

7. Tsiaflakis, P.; Diehl, M.; Moonen, M. Distributed Spectrum Management Algorithms for Multiuser DSL Networks. IEEE Transactions on Signal Processing 2008, 56, 4825-4843. doi:10.1109/TSP.2008.927460.

8. Guenach, M.; Louveaux, J.; Vandendorpe, L.; Whiting, P.; Maes, J.; Peeters, M. On Signal-to-Noise Ratio-Assisted Crosstalk Channel Estimation in Downstream DSL Systems. IEEE Transactions on Signal Processing 2010, 58, 2327-2338. doi:10.1109/TSP.2010.2040408.

9. Broadband Forum TR-320. Techniques to Mitigate Uncancelled Crosstal on Vectored VDSL2 Lines. Technical report, Broadband Forum, 2014.

10. Vatalaro, F.; Mazzenga, F.; Giuliano, R. The Sub-Band Vectoring Technique for Multi-Operator Environments. IEEE Access 2016, 4, 3310-3321. doi:10.1109/ACCESS.2016.2580198.

11. Group, E.R. Bitstream Access, ERG Consultation Document, 2003.

12. Group, E.R. Bitstream Access, ERG Common Position. https://www.berec.europa.eu/doc/whatsnew/ erg_0333rev1_bitstream_access_common_position.pdf, April 2nd 2004. [Online; accessed 5-March-2019].

13. Ruffini, M.; Payne, D.; Giglio, A.D.; Graudszus, W.; Montalvo, J.; Wosinska, L. Final report on regulation, policy and multi-business model usaged.

14. Plückebaum, T.; Jay, S.; Neumann, K.H. VDSL and G. fast Vectoring and the impact on VULA, 2014.

15. S. Beardsley, L. Enriquez, M.G.S.S. Creating a Fiber Future: The Regulatory Challenge. Technical report, McKinsey \& Company Inc., 2011.

16. M. Ruffini, D.P. Business and ownership model case studies for next generation FTTH deployment. Technical report, DISCUS EU project whitepaper, 2016.

17. D.B. Payne, M.R. Local Loop Unbundling regulation: is it a barrier to FTTH deployment? Technical report, DISCUS EU project whitepaper, 2016.

18. Peterson, L.; Al-Shabibi, A.; Anshutz, T.; Baker, S.; Bavier, A.; Das, S.; Hart, J.; Palukar, G.; Snow, W. Central office re-architected as a data center. IEEE Communications Magazine 2016, 54, 96-101. doi:10.1109/MCOM.2016.7588276.

19. Ruffini, M.; Achouche, M.; Arbelaez, A.; Bonk, R.; Di Giglio, A.; Doran, N.J.; Furdek, M.; Jensen, R.; Montalvo, J.; Parsons, N.; Pfeiffer, T.; Quesada, L.; Raack, C.; Rohde, H.; Schiano, M.; Talli, G.; Townsend, P.; Wessaly, R.; Wosinska, L.; Yin, X.; Payne, D.B. Access and metro network convergence for flexible end-to-end network design [invited]. IEEE/OSA Journal of Optical Communications and Networking 2017, 9, 524-535. doi:10.1364/JOCN.9.000524.

20. Alvarez, P.; Marchetti, N.; Payne, D.; Ruffini, M. Backhauling mobile systems with XG-PON using grouped assured bandwidth. 2014 19th European Conference on Networks and Optical Communications - (NOC), 2014, pp. 91-96. doi:10.1109/NOC.2014.6996834.

21. Shibata, N.; Tashiro, T.; Kuwano, S.; Yuki, N.; Terada, J.; Otaka, A. Mobile front-haul employing ethernet-based TDM-PON system for small cells. 2015 Optical Fiber Communications Conference and Exhibition (OFC), 2015. doi:10.1364/OFC.2015.M2J.1.

22. Fiorani, M.; Skubic, B.; Mårtensson, J.; Valcarenghi, L.; Castoldi, P.; Wosinska, L.; Monti, P. On the design of 5G transport networks. Photonic Network Communications 2015, 30. doi:10.1007/s11107-015-0553-8.

23. Szcześniak, I.; Pach, A.R.; Woźna-Szcześniak, B. Performance of interoperator fixed-mobile network sharing. 2017 International Conference on Optical Network Design and Modeling (ONDM), 2017.

24. Cornaglia, B.; Young, G.; Marchetta, A. Fixed Access Network Sharing. Optical Fiber Technology 2015, 26, Part A. Next Generation Access Networks, doi:10.1016/j.yofte.2015.07.008.

25. Jaber, M.; Imran, M.A.; Tafazolli, R.; Tukmanov, A. 5G Backhaul Challenges and Emerging Research Directions: A Survey. IEEE Access 2016, 4. doi:10.1109/ACCESS.2016.2556011.

26. Reumerman, H.; Ruffini, M. Distributed power control for reliable broadcast in inter-vehicle communication systems. 2005. 2nd International Workshop on Intelligent Transportation, 2005.

27. Ruffini, M.; Reumerman, H.. Power-rate adaptation in high-mobility distributed ad-hoc wireless networks. 2005 IEEE 61st Vehicular Technology Conference, 2005, Vol. 4, pp. 2299-2303 Vol. 4. doi:10.1109/VETECS.2005.1543745. 
28. Lianghai, J.; Liu, M.; Weinand, A.; Schotten, H.D. Direct vehicle-to-vehicle communication with infrastructure assistance in 5G network. 2017 16th Annual Mediterranean Ad Hoc Networking Workshop (Med-Hoc-Net), 2017, pp. 1-5. doi:10.1109/MedHocNet.2017.8001639.

29. Arena, F.; Pau, G. An Overview of Vehicular Communications. Future Internet $2019,11$. doi:10.3390/fi11020027.

30. Taniguchi, T.; Kobayashi, T.; Kuwano, S.; i. Kani, J.; Terada, J.; Kimura, H. Mobile optical network for future radio access. 2014 12th International Conference on Optical Internet 2014 (COIN), 2014. doi:10.1109/COIN.2014.6950589.

31. Tashiro, T.; Kuwano, S.; Terada, J.; Kawamura, T.; Tanaka, N.; Shigematsu, S.; Yoshimoto, N. A novel DBA scheme for TDM-PON based mobile fronthaul. OFC 2014, 2014. doi:10.1364/OFC.2014.Tu3F.3.

32. Ou, H.; Kobayashi, T.; Shimada, T.; Hisano, D.; Terada, J.; Otaka, A. Passive optical network range applicable to cost-effective mobile fronthaul. 2016 IEEE International Conference on Communications (ICC), 2016. doi:10.1109/ICC.2016.7511482.

33. Zhou, S.; Liu, X.; Effenberger, F.; Chao, J. Mobile-PON: A High-Efficiency Low-Latency Mobile Fronthaul Based on Functional Split and TDM-PON with a Unified Scheduler. Optical Fiber Communication Conference. Optical Society of America, 2017. doi:10.1364/OFC.2017.Th3A.3.

34. Rawshan, F.; Ju, M.; Park, Y. Application of a multi-OLT PON system and its dynamic bandwidth allocation algorithm. 2012 Fourth International Conference on Ubiquitous and Future Networks (ICUFN), 2012. doi:10.1109/ICUFN.2012.6261710.

35. Li, C.; Guo, W.; Wang, W.; Hu, W.; Xia, M. Bandwidth resource sharing on the XGPON transmission convergence layer in a multi-operator scenario. IEEE/OSA Journal of Optical Communications and Networking 2016, 8. doi:10.1364/JOCN.8.000835.

36. Li, C.; Guo, W.; Wang, W.; Hu, W.; Xia, M. PON bandwidth resource sharing schemes in a multi-operator scenario. 2017 International Conference on Computing, Networking and Communications (ICNC), 2017. doi:10.1109/ICCNC.2017.7876161.

37. Elrasad, A.; Afraz, N.; Ruffini, M. Virtual Dynamic Bandwidth Allocation Enabling True PON Multi-Tenancy. Optical Fiber Communication Conference. Optical Society of America, 2017. doi:10.1364/OFC.2017.M3I.3.

38. Mickelsson, H. WDM-PON in mobile backhaul. 2011 13th International Conference on Transparent Optical Networks, 2011. doi:10.1109/ICTON.2011.5970770.

39. Weis, E.; Breuer, D.; Krauß, S. Assessment of fixed mobile converged backhaul and fronthaul networks. 2016 18th International Conference on Transparent Optical Networks (ICTON), 2016. doi:10.1109/ICTON.2016.7550661.

40. Theodoras, J. WDM-PON: A New Access Architecture for a New Bandwidth Landscape. BROADBAND PROPERTIES 2009.

41. Carmona, D.; Prat, J.; Tomkos, I.; Marco, M.P.S. GPON unbundling for multioperator access. 2012 15th International Telecommunications Network Strategy and Planning Symposium (NETWORKS), 2012. doi:10.1109/NETWKS.2012.6381701.

42. Luo, Y.; Zhou, X.; Effenberger, F.; Yan, X.; Peng, G.; Qian, Y.; Ma, Y. Time- and Wavelength-Division Multiplexed Passive Optical Network (TWDM-PON) for Next-Generation PON Stage 2 (NG-PON2). Journal of Lightwave Technology 2013, 31, 587-593. doi:10.1109/JLT.2012.2215841.

43. Hossen, M.; Hanawa, M. Multi-OLT and multi-wavelength PON-based open access network for improving the throughput and quality of services. Optical Switching and Networking 2015, 15. doi:10.1016/j.osn.2014.09.001.

44. Iida, D.; Kuwano, S.; ichi Kani, J.; Terada, J. Dynamic TWDM-PON for Mobile Radio Access Networks. Opt. Express 2013, 21, 26209-26218. doi:10.1364/OE.21.026209.

45. Sherwood, R.; Gibb, G.; Yap, K.K.; Appenzeller, G.; Casado, M.; McKeown, N.; Parulkar, G. Flowvisor: A network virtualization layer. OpenFlow Switch Consortium, Tech. Rep 2009.

46. Yu, M.; Yi, Y.; Rexford, J.; Chiang, M. Rethinking virtual network embedding: substrate support for path splitting and migration. ACM SIGCOMM Computer Communication Review 2008, 38.

47. Argyropoulos, C.; Mastorakis, S.; Giotis, K.; Androulidakis, G.; Kalogeras, D.; Maglaris, V. Control-plane slicing methods in multi-tenant software defined networks. Integrated Network Management (IM), 2015 IFIP/IEEE International Symposium on. IEEE, 2015. 
48. Drutskoy, D.; Keller, E.; Rexford, J. Scalable network virtualization in software-defined networks. IEEE Internet Computing 2013, 17.

49. Bozakov, Z.; Papadimitriou, P. Autoslice: automated and scalable slicing for software-defined networks. Proceedings of the 2012 ACM conference on CoNEXT student workshop. ACM, 2012.

50. Muñoz, R.; Vilalta, R.; Casellas, R.; Martínez, R.; Szyrkowiec, T.; Autenrieth, A.; López, V.; López, D. $\mathrm{SDN} / \mathrm{NFV}$ orchestration for dynamic deployment of virtual SDN controllers as VNF for multi-tenant optical networks. Optical Fiber Communications Conference and Exhibition (OFC), 2015. IEEE, 2015.

51. Vilalta, R.; Mayoral, A.; Munoz, R.; Casellas, R.; Martínez, R. Multitenant transport networks with SDN/NFV. Journal of Lightwave Technology 2016, 34.

52. Kerpez, K.J.; Cioffi, J.M.; Ginis, G.; Goldburg, M.; Galli, S.; Silverman, P. Software-defined access networks. IEEE Communications magazine 2014, 52.

53. Clegg, R.G.; Spencer, J.; Landa, R.; Thakur, M.; Mitchell, J.; Rio, M. Pushing software defined networking to the access. Software Defined Networks (EWSDN), 2014 Third European Workshop on. IEEE, 2014.

54. XOS - Unleash the Power of OpenStack. http:/ / xosproject.org/. (Accessed on 02/14/2017).

55. Home » OpenStack Open Source Cloud Computing Software. https://www.openstack.org/. (Accessed on 02/14/2017).

56. Alvarez, P.; Marchetti, N.; Ruffini, M. Evaluating dynamic bandwidth allocation of virtualized passive optical networks over mobile traffic traces. IEEE/OSA Journal of Optical Communications and Networking 2016, 8, 129-136. doi:10.1364/JOCN.8.000129.

57. Elrasad, A.; Ruffini, M. Frame Level Sharing for DBA virtualization in multi-tenant PONs. 2017 International Conference on Optical Network Design and Modeling (ONDM), 2017, pp. 1-6. doi:10.23919/ONDM.2017.7958528.

58. Ruffini, M.; Slyne, F. Moving the Network to the Cloud: The Cloud Central Office Revolution and Its Implications for the Optical Layer. Journal of Lightwave Technology 2019, 37, 1706-1716. doi:10.1109/JLT.2019.2891990.

59. Slyne, F.; Elrasad, A.; Bluemm, C.; Ruffini, M. Demonstration of Real Time VNF Implementation of OLT with Virtual DBA for Sliceable Multi-Tenant PONs. 2018 Optical Fiber Communications Conference and Exposition (OFC), 2018, pp. 1-3.

60. Slyne, F.; Giller, R.; Singh, J.; Ruffini, M. Experimental Demonstration of DPDK Optimised VNF Implementation of Virtual DBA in a Multi-Tenant PON. 2018 European Conference on Optical Communication (ECOC), 2018, pp. 1-3. doi:10.1109/ECOC.2018.8535109.

61. Slyne, F.; Cornaglia, B.; Boselli, M.; Ruffini, M. 3-stage Hierarchical Quality of Service for Multi-tenant Passive Optical Network. 2019, 23rd Conference on Optical Network Design and Modeling - (ONDM), 2019.

62. Slyne, F.; Cornaglia, B.; Boselli, M.; Ruffini, M. Single-stage scheduler for accurate QoS delivery in virtualised multi-tenant Passive Optical Networks. 2019, European Conference on Optical Communications - (ECOC), 2019.

63. of European Regulators for Electronic Communications (BEREC), B. BEREC Report on the New Forms of Sharing Passive Optical Networks Based on Wavelength Division Multiplexing, 2017.

64. Markendahl, J.; Ghanbari, a.; Mölleryd, B.G. Network cooperation between mobile operators : why and how competitors cooperate? IMP Conference 2103 :, 2013. QC 20140325.

65. GABRIEL, C. MOBILE OPERATOR CAPEX SPENDING: WORLDWIDE TRENDS AND FORECASTS 2016-2025. Analysys Mason 2017.

66. Rendon Schneir, J.; Xiong, Y. Cost analysis of network sharing in FTTH/PONs. IEEE Communications Magazine 2014, 52, 126-134. doi:10.1109/MCOM.2014.6871680.

67. Khan, A.; Kellerer, W.; Kozu, K.; Yabusaki, M. Network sharing in the next mobile network: TCO reduction, management flexibility, and operational independence. IEEE Communications Magazine 2011, 49, $134-142$. doi:10.1109/MCOM.2011.6035827.

68. Grijpink, F.; Ménard, A.; Sigurdsson, H.; Vucevic, N. Network sharing and 5G: A turning point for lone riders, 2018.

69. Lange, C.; Breuer, D.; Huelsermann, R. Cost effectiveness of site reduction in optical access network: A CapEx based comparison of different technologies. 2009 11th International Conference on Transparent Optical Networks, 2009, pp. 1-4. doi:10.1109/ICTON.2009.5185100. 
70. Results of the 3.6 GHz Band Spectrum Award, 2017.

71. Mishra, V.; Mathew, J.; Lau, C.T. QoS and Energy Management in Cognitive Radio Network: Case Study Approach; Springer, 2016.

72. Ghasemi, A.; Sousa, E.S. Spectrum sensing in cognitive radio networks: requirements, challenges and design trade-offs. IEEE Communications Magazine 2008, 46, 32-39. doi:10.1109/MCOM.2008.4481338.

73. Secondary Markets for Spectrum, 2005. doi:https://doi.org/https://doi.org/10.1787/232354100386.

74. Afraz, N.; Elrasad, A.; Ruffini, M. DBA Capacity Auctions to Enhance Resource Sharing across Virtual Network Operators in Multi-Tenant PONs. 2018 Optical Fiber Communications Conference and Exposition (OFC), 2018, pp. 1-3.

75. Afraz, N.; Ruffini, M. A Marketplace for Real-time Virtual PON Sharing. 2018 Asia Communications and Photonics Conference (ACP), 2018, pp. 1-3. doi:10.1109/ ACP.2018.8596221.

76. Afraz, N.; Ruffini, M. A Sharing Platform for Multi-Tenant PONs. Journal of Lightwave Technology 2018, 36, 5413-5423. doi:10.1109/JLT.2018.2875188.

77. Christidis, K.; Devetsikiotis, M. Blockchains and Smart Contracts for the Internet of Things. IEEE Access 2016, 4, 2292-2303. doi:10.1109/ACCESS.2016.2566339.

78. Backman, J.; Yrjölä, S.; Valtanen, K.; Mämmelä, O. Blockchain network slice broker in 5G: Slice leasing in factory of the future use case. 2017 Internet of Things Business Models, Users, and Networks, 2017, pp. 1-8. doi:10.1109/CTTE.2017.8260929.

79. Afraz, N.; Ruffini, M. A Distributed Bilateral Resource Market Mechanism for Future Telecommunications Networks. 2019 IEEE Globecom Workshops (GC Wkshps): IEEE GLOBECOM 2019 Workshop on Telecommunications and Blockchain (GC 2019 Workshops - TB); , 2019.

80. Borjigin, W.; Ota, K.; Dong, M. In Broker We Trust: A Double-Auction Approach for Resource Allocation in NFV Markets. IEEE Transactions on Network and Service Management 2018, 15, 1322-1333. doi:10.1109/TNSM.2018.2882535.

81. Jiang, L.; Wang, Q.; Song, R.; Ye, B.; Dai, J. A Truthful Double Auction Framework for Promoting Femtocell Access. IEEE Access 2019, 7, 34991-35000. doi:10.1109/ACCESS.2019.2904548.

82. Liu, Y.; Xu, X.; Pan, J.; Zhang, J.; Zhao, G. A Truthful Auction Mechanism for Mobile Crowd Sensing With Budget Constraint. IEEE Access 2019, 7, 43933-43947. doi:10.1109/ACCESS.2019.2902882.

83. Wang, Q.; Huang, J.; Chen, Y.; Wang, C.; Xiao, F.; Luo, X. PROST : Privacy-Preserving and Truthful Online Double Auction for Spectrum Allocation. IEEE Transactions on Information Forensics and Security 2019, 14, 374-386. doi:10.1109/TIFS.2018.2850330.

84. Davim, J.P.; Pinto, A.N. CAPEX Model for PON Technology. 2010 2nd International Conference on Evolving Internet, 2010, pp. 192-196. doi:10.1109/INTERNET.2010.41.

85. Macaluso, I.; Cornaglia, B.; Ruffini, M. Antenna, spectrum and capacity trade-off for cloud-RAN Massive distributed MIMO over next generation PONs. 2017 Optical Fiber Communications Conference and Exhibition (OFC), 2017, pp. 1-3. 